\title{
Solitary Waves of Planar Ferromagnets and the Breakdown of the Spin-Polarized Quantum Hall Effect
}

\author{
N.R. Cooper \\ Institut Laue-Langevin, Avenue des Martyrs, B.P. 156, 38042 Grenoble, France \\ and T.C.M. Group, Cavendish Laboratory, Madingley Road, Cambridge CB3 0HE, United Kingdom.
}

(28 December, 1997)

\begin{abstract}
A branch of uniformly-propagating solitary waves of planar ferromagnets is identified. The energy dispersion and structures of the solitary waves are determined for an isotropic ferromagnet as functions of a conserved momentum. With increasing momentum, their structure undergoes a transition from a form ressembling a droplet of spin-waves to a Skyrmion/anti-Skyrmion pair. An instability to the formation of these solitary waves is shown to provide a mechanism for the electric field-induced breakdown of the spin-polarized quantum Hall effect.
\end{abstract}

PACS numbers: 75.10.Hk, 11.27.+d, 73.40.Hm

Continuum descriptions of the static and dynamic properties of ferromagnets give rise to many forms of nonlinear structure [1]. These structures depend strongly on the symmetries and dimensionality of the ferromagnet. In two dimensions, the existence of a topological classification of spin-configurations [2] has important consequences. In particular, the topological index plays a key role in the construction of a sequence of static "Skyrmion" solitons of an isotropic ferromagnet [3]. These solitons have been of recent interest in quantum Hall systems, for which the low-energy charged excitations can be described as Skyrmions [A].

The topological density is of particular importance for the dynamical properties of planar ferromagnets. It has been established that the dynamics are contrained not only by the existence of the topological index, but also by conservation laws based on moments of the topological density [5]. Here, we use the properties of a conserved momentum, defined in terms of the dipole moment of the topological density, to identify a branch of uniformly-propagating solitary waves of planar ferromagnets. At large momentum the solitary waves appear as Skyrmion/anti-Skyrmion pairs, which move at a constant velocity perpendicular to the line separating their centres. To the best of our knowledge, these are the first propagating solitary waves of a planar ferromagnet to be identified. We further show that an instability to the creation of these solitary waves leads to a breakdown of the spin-polarized quantum Hall effect, akin to the destruction of superfluid flow by vortex-ring nucleation [6].

We start from the dissipationless Landau-Lifshitz equation, which provides a semiclassical description of the dynamics of the local magnetization of a ferromagnet, as represented by the 3 -component unit vector $n_{i}(\boldsymbol{r}, t)[7]$

$$
\rho J \frac{\partial n_{i}}{\partial t}=-\epsilon_{i j k} n_{j} \frac{\delta E}{\delta n_{k}} .
$$

$\rho$ is the density of magnetic moments, each of angular momentum $J$, and $E\left(n_{i}, \nabla_{\alpha} n_{j}\right)$ is an energy functional of the magnetization and its spatial derivatives. (Summa- tion convention is assumed throughout, the labels $i, j, k$ running over the three spin-components and $\alpha, \beta$ over the two spatial dimensions.)

The total energy, $E$, and the modulus, $n_{i}^{2}$, are conserved by Eq.(11). There are several other conserved quantities that are important in what follows: the topological index, $Q$, the linear momentum, $P_{\alpha}$, and the total number of spin-reversals, $N$, defined by [5]

$$
\begin{aligned}
Q & \equiv \int d^{2} \boldsymbol{r} q(\boldsymbol{r}), \\
P_{\alpha} & \equiv 4 \pi \rho J \epsilon_{\alpha \beta} \int d^{2} \boldsymbol{r} r_{\beta} q(\boldsymbol{r}), \\
N & \equiv \rho J / \hbar \int d^{2} \boldsymbol{r}\left(1-n_{z}\right),
\end{aligned}
$$

where $q(\boldsymbol{r}) \equiv \frac{1}{8 \pi} \epsilon_{i j k} \epsilon_{\alpha \beta} n_{i} \nabla_{\alpha} n_{j} \nabla_{\beta} n_{k}$ is the topological density.

We will focus on configurations for which the magnetization tends to $n_{z}=1$ at spatial infinity. In this case, the topological index $Q$ is an integer [2,3], that is conserved under smooth deformations of $n_{i}(\boldsymbol{r})$. $Q$ is therefore a constant of any regular dynamics. The conservation of $P_{\alpha}$ and $N$ under Eq. (11) requires certain restrictions on the terms in the energy functional. It is sufficient that the energy functional describe a translationally-invariant ferromagnet, with at most uniaxial anisotropy (chosen along the $\hat{z}$-axis), and that magnetic dipolar interactions can be neglected [5]. These conditions are met by the energy functionals considered below.

Essential to our work is the definition of linear momentum (3) in terms of the dipole moment of the topological density. This form avoids ambiguities inherent in other definitions [1] (see Ref. [5]), that would prove important for the configurations studied below. We use the conservation of $P_{\alpha}, N$ and $Q$ to establish the existence of a branch of solitary waves, following a method that has been used to construct solitons of the 1-dimensional Heisenberg ferromagnet [8]. 
Consider the spin-configurations that minimize the energy $E$ for given values of $P_{\alpha}, N$, and $Q$. By combining the variational equations with Eq. (11) one finds that these spin-configurations have the time evolution

$$
\frac{\partial n_{i}}{\partial t}=-v_{\alpha} \nabla_{\alpha} n_{i}-\omega \epsilon_{i j k} n_{j} \hat{z}_{k}
$$

where $v_{\alpha}$ and $\omega$ are Lagrange multipliers introduced to enforce the constaints on $P_{\alpha}$ and $N$. This equation shows that the extremal spin-configurations are travelling waves, that translate at a uniform velocity $v_{\alpha}$ while the magnetization precesses around the $\hat{\boldsymbol{z}}$ axis at an angular frequency $\omega$. Defining an "energy dispersion" $E_{Q}^{*}\left(P_{\alpha}, N\right)$ by the minimum energy at given $P_{\alpha} N$ and $Q$, the variational equations further require

$$
v_{\alpha}=\left.\frac{\partial E_{Q}^{*}}{\partial P_{\alpha}}\right|_{N} ; \quad \omega=-\left.\frac{1}{\hbar} \frac{\partial E_{Q}^{*}}{\partial N}\right|_{P_{\alpha}} .
$$

Whilst one can imagine searching for such travelling waves within each topological subspace, note that the momentum (3) transforms as $P_{\alpha} \rightarrow P_{\alpha}-Q R_{\alpha}$ un$\operatorname{der} n_{i}\left(r_{\alpha}\right) \rightarrow n_{i}\left(r_{\alpha}+R_{\alpha}\right)$. Consequently, for a ferromagnet that is translationally-invariant, $E_{Q \neq 0}^{*}(P, N)=$ $E_{Q \neq 0}^{*}(0, N)$ and all modes have vanishing velocity (6). For the $Q=0$ sector, however, one does expect the energy of a translationally-invariant ferromagnet to depend on the momentum. In the following, we will study the properties of a translationally-invariant ferromagnet within this $Q=0$ subspace (henceforth we drop the subscript on $\left.E_{Q}^{*}\right)$. Amongst the extremal spin-configurations that determine $E^{*}\left(P_{\alpha}, N\right)$, we will find configurations that are localized in space and have non-zero $v_{\alpha}$ and $\omega$. These are therefore uniformly-propagating solitary waves [2] of the ferromagnet.

Specifically, we study an isotropic ferromagnet, for which the energy functional for long-wavelength distortions is the $O(3)$ non-linear $\sigma$-model

$$
E^{n l \sigma}\left[n_{i}, \nabla_{\alpha} n_{j}\right] \equiv \frac{1}{2} \rho_{s} \int d^{2} \boldsymbol{r}\left(\nabla_{\alpha} n_{i}\right)^{2},
$$

characterized by the spin-stiffness $\rho_{s}$. Our results also apply to extensions of this model involving additional terms that are functions of $Q, N$ and $P_{\alpha}$ (these simply shift the velocities and precession frequencies of the modes we construct). In the following, we will work with units such that $\rho_{s}=\rho J=\hbar=1$.

We have investigated the energy dispersion $E^{*}\left(P_{\alpha}, N\right)$ of the non-linear $\sigma$-model numerically, using a lattice discretization of the continuum ferromagnet (details will be presented elsewhere [9]). The results we present are for a square region of $249 \times 249$ spins, bounded by spins fixed to $n_{z}=1$. The parameters are chosen such that the results well represent the continuum theory (7) defined within a square region (of side $L$, say). The scaleinvariance of such a theory requires that $E^{*}(P, N, L)=$ $E^{*}\left(P \lambda, N \lambda^{2}, L \lambda\right)$ for all scale-factors $\lambda$, so the energy can depend only on the "scaled momentum" $p \equiv P / \sqrt{N}$, and the "boundary parameter" $\eta \equiv N / L^{2}$. The properties of an infinite system are determined by considering the limit $\eta \rightarrow 0$.

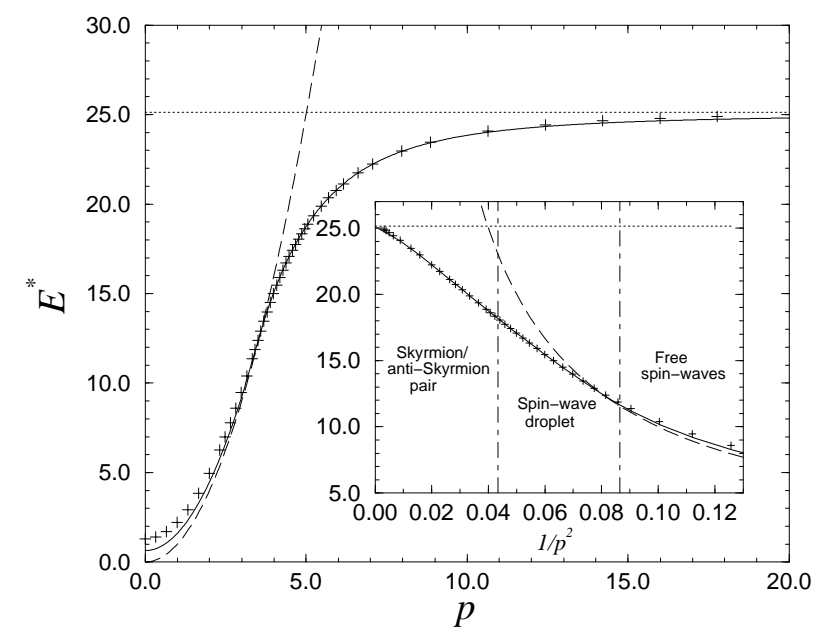

FIG. 1. The energy dispersion of the isotropic ferromagnet, for $\eta=0.032$ (solid line) and $\eta=0.064$ (crosses). The energies, for an infinite system, of free spin-waves (dashed line) and a non-interacting Skyrmion/anti-Skyrmion pair (dotted line) are shown for comparison. Inset: the same quantities as functions of $1 / p^{2}$, indicating the three regimes of the $\eta \rightarrow 0$ limit.

Figure 1 shows results for the minimum energy as a function of $p$ at two values of the boundary parameter $\eta$. Although weak dependences on $\eta$ do remain, it is clear that the energy dispersion $E^{*}(p, \eta)$ is tending to a smooth function as $\eta \rightarrow 0$. The limiting configurations will describe travelling waves with non-zero velocity and precession frequency (6).

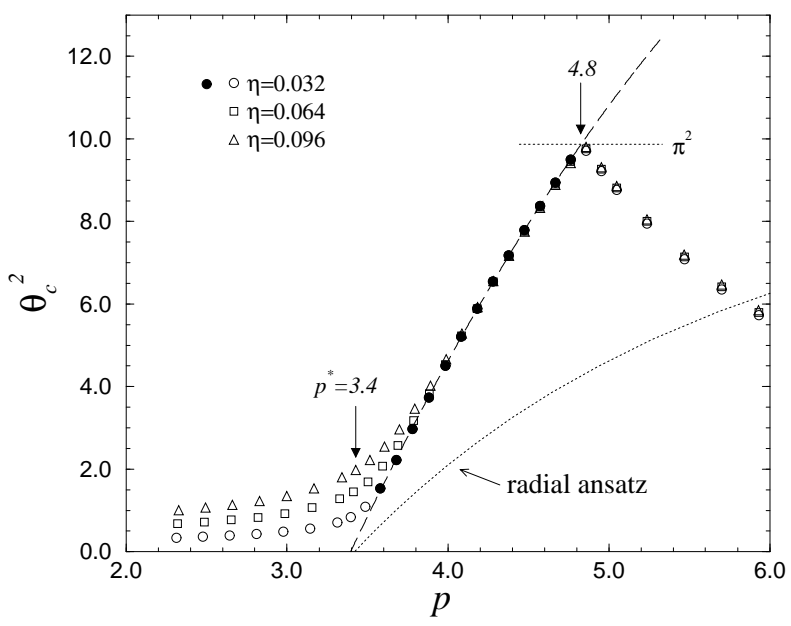

FIG. 2. Variation of the square of the polar angle at the centre of the system with scaled momentum. An extrapolation, using the filled circles, suggests that as $\eta \rightarrow 0, \theta_{c}$ would vanish for $p<3.4$. Also shown are the results for an ansatz described in the text. 
A careful study of the spin-configurations shows that for $\eta \rightarrow 0$, there is a transition from spatially-extended configurations at small $p$, to spatially-localized configurations at large $p$. This is illustrated in Figure 2, which shows the (square of the) polar angle at the centre of the system $\theta_{c}$ as a function of $p$ for several values of $\eta$. For $p \gtrsim 3.5, \theta_{c}$ is weakly-dependent on $\eta$, and can be assumed to represent well an unbounded system. For $p \lesssim 3.5, \theta_{c}^{2}$ decreases steadily down to the smallest values of $\eta$ studied, showing that the configurations remain sensitive to the presence of the boundaries. An extrapolation indicates that $\theta_{c}^{2}$ would vanish at a scaled momentum of $p^{*}=3.4$ (the dashed line in Fig. 2).

Within the assumption that $\theta_{c}$ does vanish as $\eta \rightarrow 0$ for $p<p^{*}$, the properties in this regime can be understood within a linearized continuum theory, in which all quantities are expanded to second-order in the polar angle $\theta(\boldsymbol{r})$. The linear theory has extremal solutions $\theta(\boldsymbol{r})=\theta_{0} \cos (\pi x / L) \cos (\pi y / L), \phi(\boldsymbol{r})=k y$, which lead to an energy dispersion $E_{S W}(p, \eta)=p^{2}+2 \pi^{2} \eta$. The energies of these configurations coincide with the energy of $N$ non-interacting spin-waves confined to a square of side $L$. We refer to them as "free spin-wave" states. The numerical results for $p<p^{*}$ are consistent with the minimalenergy spin-configurations of the infinite system being free spin-wave states: the spin-configurations ressemble those of the linearized continuum theory, and their energies approach the free spin-wave energies of an infinite system continuously as $\eta \rightarrow 0$ (see Fig. 1).

In contrast, the spin-configurations above the transition, $p>p^{*}$, cannot be described within the linearized theory. In this regime, $\theta_{c}$ tends to a finite value as $\eta \rightarrow 0$, and the spin-configurations are spatially-localized. These configurations constitute the branch of propagating solitary waves that forms the main subject of the present paper. (a)

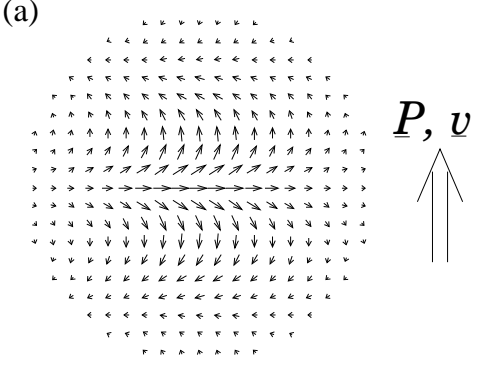

(b)

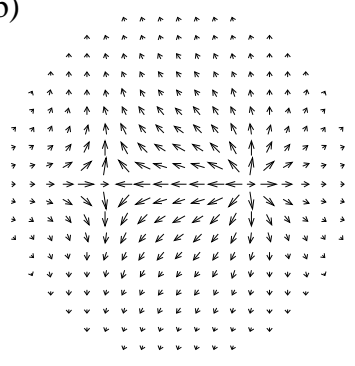

FIG. 3. Spin-configurations of the solitary waves at (a) $p=3.64$, and (b) $p=12.4$. The vectors show the projection of the spin onto the $x y$-plane for those spins with $n_{z}<0.98$ (for clarity only one spin in six is plotted). The direction of the momentum and velocity of the solitary waves is indicated.

Typical spin-configurations of these solitary waves are shown in Fig. 3. For small momenta (yet larger than $p^{*}$ ) the configurations ressemble the free-spin-wave states described above, but are localized in space [Fig. 3(a)].
We refer to these configurations as "spin-wave droplets". For large momenta, two vortices of opposite sign appear in the projection of the spins on the $x y$ plane [Fig. 3(b)]. In fact, such spin-configurations contain no singularity, since the vortices occur around points at which $n_{z}=-1$, as occurs in (anti-)Skyrmion configurations [3]. Accordingly, we refer to these configurations as "Skyrmion/anti-Skyrmion pairs". The transition between spin-wave droplets and Skyrmion/anti-Skyrmion pairs is found to occur at $p=4.8$ (see Fig. 2). Note that this transition is effected by continuous deformations of the spin-configuration. The spin-wave droplet and Skyrmion/anti-Skyrmion pair configurations therefore form part of the same branch of solitary waves; the differing names we have assigned indicate only the differences in the forms of the spin-configuration.

As the scaled momentum increases beyond $p=4.8$, the separation between the Skyrmion and anti-Skyrmion grows continuously and the energy approaches the energy, $8 \pi$, of a non-interacting Skyrmion/anti-Skyrmion pair [3]. Asymptotic forms that have been proposed for a widely-separated Skyrmion/anti-Skyrmion pair [10] have a number of spin-reversals $N$ that diverges logarithmically with system size, and so correspond to the scaled momentum $p=0$. However, deformed versions of these configurations can be expected to lead to asymptotic interactions of the form $\sim 1 /\left[p^{2} \log (p)\right][9]$. We find an approximately $1 / p^{2}$ dependence (see the inset to Fig. 1). Logarithmic corrections cannot be studied, owing to finite-size effects that appear once the Skyrmion/antiSkyrmion separation $\sim P / 4 \pi$ becomes comparable to the system size $L$ (i.e. when $p \gtrsim 4 \pi / \sqrt{\eta}$ ).

Previous numerical studies [11] of the timedevelopment of a spin-configuration ressembling Fig. B(b) under a Landau-Lifshitz dynamics showed evidence for a uniform drift parallel to the momentum. The present work establishes that solitary waves of this form exist for the non-linear $\sigma$-model, and determines their structures and velocities.

Having described the properties of the free spin-waves and solitary waves, we return briefly to discuss the transition at $p^{*}=3.4$ that was inferred by the extrapolation shown in Fig. 2. Further evidence that a transition occurs is obtained by making a radially-symmetric ansatz for the spin-wave droplet states: $\theta(\boldsymbol{r})=f(|\boldsymbol{r}|), \phi=k y$. The non-linear equation for $f(r)$ that arises from extremizing the energy has been studied in another context [12]. In the present context, the results indicate that localized solutions are lost below $p_{\text {rad }}^{*}=3.4$ (see Fig. 2). The existence of this transition lends weight to the idea that a transition occurs in the unrestricted case. Furthermore, the coincidence of the two transition points, $p^{*}=p_{\text {rad }}^{*}$, suggests that the configuration at the transition for the unrestricted problem is of the form assumed in the radially-symmetric ansatz.

We now turn to discuss the application of these results to quantum Hall ferromagnets (QHFs). These are the incompressible states responsible for the quantized 
Hall effect of two-dimensional electron systems at certain filling fractions 13,4]. The long-wavelength dynamics of QHFs are described by the Landau-Lifshitz equation 44,14, with the Zeeman energy, $\left(g \mu_{B} B\right) N$, and the nonlinear $\sigma$-model term (7) as the leading terms in a gradient expansion of the energy functional [15]. We will adopt this level of approximation, allowing the results presented above to be applied directly. Higher-order terms in the gradient expansion (e.g. long-range Coulomb forces [4]) will not affect the qualitative discussion [9].

Our results provide a semiclassical description of the neutral excitations of a $\mathrm{QHF}$ involving $N$ spin-reversals with total momentum $\boldsymbol{P}$ (this description is accurate in the limit $N \gg 1$ ). The nature of the low-energy excitation depends on the value of the scaled momentum $p$ : for $p<p^{*}$ it is a collection of $N$ spin-waves, each of momentum $\boldsymbol{P} / N$; for $p>p^{*}$ it is a solitary wave, ressembling a spin-wave droplet or Skyrmion/anti-Skyrmion pair. These solitary waves are highly reminiscent of the "magnetic exciton" picture of a single spin-wave [16]: two oppositely-charged particles move at a constant velocity perpendicular to the line joining their centres. Indeed, just as Skyrmions are the large-spin versions of the quasiparticles of a QHF, these dynamical solitary waves are the large-spin analogues of the spin-waves.

Quantum effects, not accessible within the semiclassical description, could lead to certain differences. If the quasiparticle gap is less than the Skyrmion gap for vanishing Zeeman energy [17, the lowest-energy states at large scaled momentum will not be Skyrmion/antiSkyrmion pairs; they will be widely-separated quasielectron/quasihole pairs. Also, if two spin-waves are weaklybound at any non-zero total momentum [18], then configurations at all values of $p$ will be spin-wave droplets. The transition found at $p=p^{*}$ will then be a crossover, becoming a true transition only in the limit $N \rightarrow \infty$.

Experimentally, one way in which the solitary waves can be accessed is through the application of an electric field $\mathcal{E}_{\alpha}$ to the QHF. For weak electric fields a quantized Hall current will flow with exponentially-small dissipation (we assume the limit of vanishing temperature). However, there exists a threshold value of the electric field [19], $\mathcal{E}_{T} \equiv \frac{1}{e \ell} \sqrt{16 \pi \rho_{s} g \mu_{B} B}$, above which the system can reduce its energy by creating single spin-waves of small momentum. The spontaneous creation of spinwaves is expected to initiate the breakdown of the quantum Hall effect 19]. Our results indicate that, as the number of spin-waves increases, one can expect spin-wave droplets and Skyrmion/anti-Skyrmion pairs to form. The appearance of Skyrmions was anticipated in a footnote to Ref. 19. . Our results provide a detailed description of the non-linear structures that can develop.

Firstly, we can determine the number of spin-waves involved in the formation of a spin-wave droplet. For a QHF placed in a field equal to the threshold field $\mathcal{E}_{T}$ (such that single spin-waves of momentum $\sqrt{g \mu_{B} B / 4 \pi \rho_{s}} \hbar / \ell$ are formed), it is energetically favourable for spin-waves to collapse to a spin-wave droplet when their number reaches $N_{c}=p^{* 2} \rho_{s} /\left(g \mu_{B} B\right)$.

Secondly, our results show that the spin-wave droplet that forms at this point is unstable to increasing its momentum (since the solitary-wave group velocity is maximal at $p=p^{*}$ ). If energy can be lost from the electron gas to the lattice, the droplet will be torn apart in the electric field, forming a Skyrmion/anti-Skyrmion pair of increasing separation (these charges will eventually reach the voltage contacts). By the continual nucleation of spinwave droplets, and their relaxation to widely-separated Skyrmion/anti-Skyrmion pairs, a d.c. current can be carried along the electric field. The quantized Hall effect is thereby destroyed, much as superfluid flow is destroyed by the nucleation and growth of vortex rings [6] (note, however, that for $\mathcal{E} \geq \mathcal{E}_{T}$ the spin-wave droplet can nucleate without thermal fluctuations).

This breakdown mechanism could most effectively be investigated in GaAs samples under hydrostatic pressure, for which the $g$-factor, and thus $\mathcal{E}_{T}$, can be made to vanish [20]. In realistic systems, disorder will affect the breakdown mechanism strongly. Most importantly, the localization of the Skyrmion and anti-Skyrmion will prevent the run-away separation expected for a clean system, and charge transport will proceed by the condensation of spin-wave droplets in the presence of pinned Skyrmion/anti-Skyrmion pairs.

I am grateful to Fabio Pistolesi, Tim Ziman and John Chalker for helpful discussions and advice.

[1] A. M. Kosevich, B. A. Ivanov, and A. S. Kovalev, Physics Reports 194, 117 (1990), and references therein.

[2] R. Rajaraman, Solitons and Instantons (North Holland, Amsterdam, 1989).

[3] A. A. Belavin and A. M. Polyakov, JETP Lett. 22, 245 (1975).

[4] S. L. Sondhi, A. Karlhede, S. A. Kivelson, and E. H. Rezayi, Phys. Rev. B 47, 16419 (1993).

[5] N. Papanicolaou and T. N. Tomaras, Nuclear Physics B 360, 425 (1991); S. Komineas and N. Papanicolaou, Physica D 99, 81 (1996).

[6] J. S. Langer and J. D. Reppy, in Progress in Low Temperature Physics, ed. C. J. Gorter, Vol. VI, Chap. 1.

[7] L. D. Landau and E. M. Lifshitz, Phys. Z. Sowjetunion 8, 153 (1935).

[8] J. Tjon and J. Wright, Phys. Rev. B 15, 3470 (1977).

[9] N. R. Cooper, (unpublished).

[10] D. Förster, Nuclear Physics B 130, 38 (1977); P. G. Sil'vestrov, Sov. J. Nucl. Phys. 51, 1121 (1990).

[11] N. Papanicolaou and W. J. Zakrzewski, Physica D 80, 225 (1995).

[12] A. S. Kovalev, A. M. Kosevich, and K. V. Maslov, JETP Lett. 30, 296 (1979). 
[13] D. H. Lee and C. L. Kane, Phys. Rev. Lett. 64, 1313 (1990).

[14] M. Stone, Phys. Rev. B 53, 16573 (1996).

[15] We reintroduce units for the QHFs: $g$ is the electron $g$-factor, $\mu_{B}$ the Bohr magneton, and $\ell \equiv \sqrt{\hbar / e B}$ the magnetic length in a field $B$.

[16] Y. A. Bychkov, S. V. Iordanskii and G. M. Eliashberg, Sov. Phys. JETP 33, 143 (1981); I. V. Lerner and Y. E.
Lozovik, Sov. Phys. JETP 51, 588 (1980).

[17] X. G. Wu and S. L. Sondhi, Phys. Rev. B 51, 14725 (1995); N. R. Cooper, Phys. Rev. B 55, R1934 (1997).

[18] M. Wortis, Phys. Rev. 132, 85 (1963).

[19] M. Rasolt, B. I. Halperin, and D. Vanderbilt, Phys. Rev. Lett. 57, 126 (1986).

[20] D. K. Maude et al., Phys. Rev. Lett. 77, 4604 (1996). 